\title{
Generalized Signal Decoupling Problem with Stability for Discrete-Time Systems ${ }^{1}$
}

\author{
F. BARbagli, ${ }^{2}$ G. MARro, ${ }^{3}$ AND D. PRATTIChizZO ${ }^{4}$
}

Communicated by G. Leitmann

\begin{abstract}
This paper deals with the decoupling problems of unknown, measurable, and previewed signals. First, well-known solutions of unknown and measurable disturbance decoupling problems are recalled. Then, new necessary and sufficient constructive conditions for the previewed signal decoupling problem are proposed. The discrete-time case is considered. In this domain, previewing a signal by $p$ steps means that the $k$ th sample of the signal to be decoupled is known $p$ steps in advance.

The main result is that the stability condition for the mentioned decoupling problems does not change; i.e., the resolving subspace to be stabilized is the same independently of the type of signal to be decoupled, no matter whether it is completely unknown, measured, or previewed.

The problem has been studied through self-bounded controlled invariants, thus minimizing the dimension of the resolving subspace which corresponds to the infimum of a lattice. The reduced dimension of the resolving controlled invariant subspace reduces the order of the controller units.
\end{abstract}

Key Words. Signal decoupling, previewed signals, geometric approach, feedforward control.

\section{Introduction}

Disturbance decoupling is a classical problem in control theory. It has been one of the first applications considered in the geometric approach

\footnotetext{
${ }^{1}$ This work was partially supported by the Italian Ministry for University and Scientific Research (MURST) and by the University of Siena Young Researcher Project.

${ }^{2}$ PhD Student, PERCRO Laboratory, Scuola Studi Superiori S. Anna, Pisa, Italy.

${ }^{3}$ Professor, Dipartimento di Elettronica, Informatica e Sistemistica, Università di Bologna, Bologna, Italy.

${ }^{4}$ Assistant Professor, Dipartimento di Ingegneria dell'Informazione, Università di Siena, Siena, Italy.
} 
framework and has been given attention for more than thirty years. In the first formulation of the disturbance decoupling problem (DDP, Refs. 1-2), disturbance signals are assumed to be unknown and unaccessible. Later, Bhattacharyya (Ref. 3) considered the so called measured signal decoupling problem (MSDP) in which the signals to be decoupled are considered measurable. The structural conditions for the MSDP to be solved are less restrictive than those for the DDP, while the stabilizability conditions are similar.

In this paper, the decoupling control problem is approached in a more general setting. Signals which are known in advance or previewed by a given amount of time are considered. Such problem will be referred to as the previewed signal decoupling problem (PSDP).

The PSDP has been investigated by Willems (Ref. 4), who derived first, in the continuous-time domain, a necessary and sufficient condition to solve the PSDP with pole placement. This solution was based on a proportionalintegral-derivative control law consisting of a feedback of the state system and a linear combination of the signal to be decoupled and its time derivative. The major drawback of these extensions of the disturbance decoupling problem in the continuous-time domain is that the control laws include distributions; hence, they are not implementable practically. Independently, Imai and Shinozuka (Ref. 5) proposed a similar necessary and sufficient condition for the PSDP with stability in both the discrete-time and continuous-time cases. In Ref. 6, Estrada and Malabre proposed a synthesis procedure to solve the PSDP problem with stability using the minimum number of required differentiators for the signal to be decoupled.

The conditions for the PSDP to be solved, given in Refs. 4-6, do not care about the dimensionality of the resolving controlled invariant subspace. Furthermore, to the best of our knowledge, the problem of reducing the dimension of the resolving subspace for the PSDP has not been investigated thoroughly in the literature. Note that using controlled invariants of minimal dimensions allows one to reduce the order of the controller units and possible state observers.

In this paper, a new solution for the PSDP with stability based on a subspace of reduced dimension is proposed. Such dimension optimization is obtained via self-bounded controlled invariants. This is a special class of the controlled invariants introduced by Basile and Marro in Refs. 7-8, which enjoys interesting properties, the most important of which is to be a lattice instead of a semilattice, hence to admit an infimum other than a supremum.

Moreover, this paper provides a unique necessary and sufficient condition for signal decoupling problems with stability independently of the type of signal to be decoupled, no matter whether it is completely unknown, 
measured, or previewed. In other terms, it is shown that the proposed resolving subspace for the PSDP problems and the well-known resolving subspace of the DDP problem proposed by Basile, Marro, and Piazzi in Ref. 9 are equivalent. The proofs are carried out in a geometric framework and are based on several lattices of self-bounded $(A, \mathscr{B})$-controlled invariants.

In this paper, discrete-time systems are considered. In such domain, the solution of the PSDP is more elegant and is implementable practically. The structure of the compensator, whereby the decoupling of the previewed signals is obtained, is discussed. It consists of a preaction unit and a postaction unit. A new synthesis procedure, based on geometric approach algorithms, is provided. Preliminary results of this work have been presented in Refs. $10-11$.

The following notation is used. $\mathbb{R}$ stands for the field of real numbers. Sets, vector spaces, and subspaces are denoted by script capital letters like $\mathscr{X}, \mathscr{T}, \mathscr{Y}$, etc. Since most of the geometric theory of the dynamic system presented herein is developed in the vector space $\mathbb{R}^{n}$, we reserve the symbol $\mathscr{X}$ for the full space; i.e., we assume $\mathscr{X}:=\mathbb{R}^{n}$. Matrices and linear maps are denoted by italic capital letters like $A, B$, etc.; the image and the null space of the generic matrix or linear transformation $A$ are denoted by im $A$ and ker $A$, respectively; the transpose of the generic real matrix $A$ is denoted by $A^{T}$, its spectrum by $\sigma(A)$, and its pseudoinverse by $A^{\#}$.

The reminder of this paper is organized as follows. Section 2 presents the structural conditions for the general PSDP. In Section 3, new necessary and sufficient conditions for the PSDP with stability are stated. In Section 4 , a synthesis procedure for the decoupling compensator is reported. Finally, in Section 5, an illustrative example is discussed.

\section{Structural Conditions for the PSDP}

Let us consider the discrete-time system

$$
\begin{aligned}
& x(k+1)=A x(k)+B u(k)+H h(k), \\
& y(k)=C x(k),
\end{aligned}
$$

where $x \in \mathscr{X}=\mathbb{R}^{n}, u \in \mathbb{R}^{m}, h \in \mathbb{R}^{h}$, and $y \in \mathbb{R}^{q}$ denote the state, the input, the signal to be decoupled, and the regulated output, respectively. In the following, the shortened notations

$$
\mathscr{B}:=\operatorname{im} B, \quad \mathscr{C}:==\operatorname{ker} C, \quad \mathscr{C}:=\operatorname{im} H
$$

will be used. 
In this paper, we deal with the signal decoupling problem when a certain degree of knowledge for the signal $h(k)$ is available. In particular, we assume that the signal $h(k)$ is previewed, i.e., it is known $p$ steps in advance, or analytically the sample $h(k)$ is known at step $k-p$. Henceforth, the signal $h(k)$ previewed by $p$ steps will be referred to as $p$-previewed $h(k)$, or shortly ${ }^{p} h(k)$. Note that a measurable disturbance can be thought as a 0 -previewed signal.

As for the MSDP, the input $B u(k)$ is used to cancel part of the (measured) disturbance, when it occurs; in the case of the PSDP, the preview is used to prepare the system dynamics to localize the signal $h(k)$ on the null space of the output matrix $C$. This is formalized in the following statement.

Problem P1. Previewed Signal Decoupling. Refer to the system (1) with zero initial condition, and assume that the input $h$ is previewed by $p$ instants of time, $p \geq 0$. Determine a control law which, making use of this preview, is able to maintain the output $y(k)$ identically zero.

In the case of nonpurely dynamic systems, i.e., when the output equation (1a) is generalized to

$$
y(k)=C x(k)+D u(k)+M h(k),
$$

the PSDP can be recast as in Problem P1 by simply inserting a dummy delay unit at the output and including it in the system equations, thus recovering an equivalent purely dynamic form.

In a geometric framework, the key tool to analyze the structural conditions for the signal decoupling problem is the well-known algorithm (Ref. 12) computing $\mathscr{S}^{*}:=\min S(A, \mathscr{C}, \mathscr{B})$, the minimal $(\mathscr{A}, \mathscr{C})$-conditioned invariant containing $\mathscr{B}$, reported here for the reader convenience:

$$
\begin{aligned}
& \mathscr{S}_{0}:==\mathscr{B}, \\
& \mathscr{S}_{i}:=\mathscr{B}+A\left(\mathscr{S}_{i-1} \cap \mathscr{C}\right) .
\end{aligned}
$$

The structural conditions to solve Problem P1 for $p$-previewed signals are given in the following theorem, whose proof can be derived easily from Refs. 4, 6 .

Theorem 2.1. The necessary and sufficient condition for Problem P1 to be solved is that

$$
\mathscr{H} \subseteq \mathscr{Y}^{*}+\mathscr{S}_{p},
$$

where $\mathscr{Y} *:=\max \mathscr{Y}(A, \mathscr{B}, \mathscr{C})$ is the maximal controlled invariant contained in the null space of $\mathscr{C}$. 
Remark 2.1. The structural condition (4) in Theorem 2.1 is similar to that proposed in Refs. 4, 6 for the continuous-time case, but is less restrictive since the condition (4) does not consider stability. It is worth noting that the case of measurable inputs is accounted for by the condition (4). In fact, measurable signals corresponds to $p=0$; therefore, (4) turns into the wellknown condition (Ref. 3)

$$
\mathscr{H} \subseteq \mathscr{Y} *+\mathscr{B} .
$$

Similarly, the lack of preview yields the well-known structural condition for unknown signals, i.e.,

$$
\mathscr{H} \subseteq \mathscr{Y}^{*} .
$$

Summarizing, since

$$
\mathscr{Y}^{*} \subseteq \mathscr{Y}^{*}+\mathscr{B} \subseteq \mathscr{Y}^{*}+\mathscr{S}_{p},
$$

the larger the preview time, the easier it is to solve the decoupling problem.

The following property characterizes the minimum number of preview steps necessary to decouple the previewed signals for a given disturbance matrix $H$.

Property Q1. Consider the system (1) and let $r$ be the minimum number of steps necessary to obtain the convergence of the algorithm for $\mathscr{S}^{*}$ [Eqs. (2)-(3)]. The minimum positive integer $p \leq r$, such that the condition (4) holds, corresponds to the minimum number of previewed steps for $h(k)$ needed to decouple the signal $H h(k)$. For $p=r$, if the condition (4) is not satisfied, the PSDP has no solution for the given disturbance matrix $H$.

If the structural condition (4) holds, it is possible to decompose the disturbance effect into two separate parts as follows:

$$
\begin{aligned}
& H=H_{V}+H_{S}, \\
& \mathscr{H}_{V}:=\operatorname{im}\left(H_{V}\right) \subseteq \mathscr{Y} * \\
& \mathscr{H}_{S}:=\operatorname{im}\left(H_{S}\right) \subseteq \mathscr{S}_{p} .
\end{aligned}
$$

The matrices $H_{V}$ and $H_{S}$ are basic to synthesize the controller solving Problem P1 as discussed in Section 4. The components of $H h(k)$ lying on $\mathscr{S}_{p}$ can be canceled through a preaction unit. For this purpose, note that $\mathscr{S}_{p}$ can be interpreted as the reachable subspace in $p, p \geq 0$, steps from $x_{0}=0$, with 
the state trajectory constrained to lie on the null space of the output matrix $C$ in the $(p-1)$-step interval $[0, p-1]$,

$$
C x(k)=0, \quad \text { for } k=0,1, \ldots p-1 .
$$

In other terms, the preaction unit, which is a part of the decoupling controller, exploits the signal preview to cancel $H_{S} h(k)$, i.e., the part of $H h(k)$ belonging to $\mathscr{S}_{p}$. Because of the special reachability subspace $\mathscr{S}_{p}$, this happens while maintaining the output identically zero. On the other hand, the signal $H_{V} h(k)$ is localized in the null space of the output matrix according to standard decoupling techniques (Ref. 12).

\section{Previewed Signal Decoupling Problem with Stability}

The $p$-previewed signal decoupling problem with stability is investigated.

Problem P2. Previewed Signal Decoupling with Stability. Refer to the system (1) with zero initial condition and assume that it is stabilizable and that the input $h$ is previewed by $p$ instants of time, $p \geq 0$. Determine a control law which, making use of the preview, is able to maintain the output $y(k)$ identically zero while keeping the state trajectory bounded.

The previewed signal decoupling with stability is approached by means of lattices of self-bounded controlled invariants (Ref. 7-8). A special attention is devoted to the dimension of the resolving subspace.

Let us introduce the lattice of all the $(A, \mathscr{B})$-controlled invariants selfbounded with respect to $\mathscr{C}$,

$$
\begin{aligned}
\Phi & =\Phi(\mathscr{B}, \mathscr{C}) \\
& =\{\mathscr{Y} \mid A \mathscr{Y} \subseteq \mathscr{Y}+\mathscr{B}, \mathscr{Y} \subseteq \mathscr{C}, \mathscr{Y} * \cap \mathscr{B} \subseteq \mathscr{Y}\},
\end{aligned}
$$

whose infimum is given by

$$
\mathscr{y} * \cap \min \mathscr{S}(A, \mathscr{C}, \mathscr{B})
$$

and the lattice of all the $\left(A, \mathscr{B}+\mathscr{H}_{V}\right)$-controlled invariants self-bounded with respect to $\mathscr{C}$,

$$
\begin{aligned}
\Phi_{1} & =\Phi\left(\mathscr{B}+\mathscr{H}_{V}, \mathscr{C}\right) \\
& =\left\{\mathscr{Y} \mid A \mathscr{Y} \subseteq \mathscr{Y}+\mathscr{B}+\mathscr{H}_{V}, \mathscr{Y} \subseteq \mathscr{C}, \mathscr{Y} * \cap\left(\mathscr{B}+\mathscr{H}_{V}\right) \subseteq \mathscr{Y}\right\},
\end{aligned}
$$


whose infimum is given by

$$
\mathscr{\mathscr { V }}_{m 1}=\mathscr{y} * \cap \min \mathscr{S}\left(A, \mathscr{C}, \mathscr{B}+\mathscr{H}_{V}\right) \text {. }
$$

The subspace $\mathscr{Y}_{m 1}$ can be written as in (13), since the condition (8) holds; therefore,

$$
\mathscr{P} * \equiv \max \mathscr{\mathscr { F }}\left(A, \mathscr{B}+\mathscr{H}_{V}, \mathscr{C}\right) .
$$

The following lemmas hold.

Lemma 3.1. The set

$$
\Phi_{2}=\left\{\mathscr{Y} \mid \mathscr{Y} \in \Phi, \mathscr{H} \subseteq \mathscr{Y}+\mathscr{S}_{p}\right\}
$$

has the following properties:

(i) is a sublattice of $\Phi$;

(ii) $\Phi_{2} \equiv\left\{\mathscr{V} \mid \mathscr{Y} \in \Phi, \mathscr{Y} * \cap\left(\mathscr{H}+\mathscr{S}_{p}\right) \subseteq \mathscr{Y}\right\}$.

\section{Proof.}

(i) We want to show that, given two generic elements $q_{1}$ and $q_{2}$ of the set $\Phi_{2}$, their sum and intersection still belongs to the same set. Such proof appears trivial for the subspace obtained by summing the two given subspaces. Let us consider now the element $/ / 1 \cap / 2$. By assumption, since both $\mathscr{Y}_{1}$ and $\mathscr{Y}_{2}$ belong to $\Phi_{2}$, it follows that

$$
\begin{aligned}
& \mathscr{H} \subseteq \mathscr{Y}_{1}+\mathscr{S}_{p}, \\
& \mathscr{H} \subseteq \mathscr{Y}_{2}+\mathscr{S}_{p},
\end{aligned}
$$

which lead to

$$
\mathscr{H} \subseteq\left(\mathscr{Y}_{1}+\mathscr{S}_{p}\right) \cap\left(\mathscr{Y}_{2}+\mathscr{S}_{p}\right) .
$$

By intersecting both terms with $\mathcal{y}^{*}+\mathscr{S}_{p}$, we obtain

$$
\mathscr{H} \subseteq\left(\left(\mathscr{Y}_{1}+\mathscr{S}_{p}\right) \cap\left(\mathscr{Y}_{2}+\mathscr{S}_{p}\right)\right) \cap\left(\mathscr{Y} *+\mathscr{S}_{p}\right),
$$

since the structural condition (4) holds, and then

$$
\mathscr{H} \subseteq\left(\mathscr{Y} * \cap\left(\mathscr{Y}_{1}+\mathscr{S}_{p}\right)\right) \cap\left(\mathscr{Y} * \cap\left(\mathscr{Y}_{2}+\mathscr{S}_{p}\right)\right)+\mathscr{S}_{p},
$$

using the distributive property, since $\mathscr{S}_{p}$ is included in $\left(\mathscr{C}_{1}+\mathscr{S}_{p}\right) \cap\left(\mathscr{C}_{2}+\mathscr{S}_{p}\right)$. Analogously, we get

$$
\mathscr{H} \subseteq\left(\left(\mathscr{C}_{1} \cap \mathscr{Y} *\right)+\left(\mathscr{Y} * \cap \mathscr{S}_{p}\right)\right) \cap\left(\left(\mathscr{C}_{2} \cap \mathscr{Y} *\right)+\left(\mathscr{Y} * \cap \mathscr{S}_{p}\right)\right)+\mathscr{S}_{p},
$$


and finally,

$$
\mathscr{H} \subseteq\left(\mathscr{Y}_{1} \cap \mathscr{Y}_{2}\right)+\mathscr{S}_{p},
$$

since $\mathscr{Y}_{1}$ and $\mathscr{Y}_{2}$ are both included in $\mathscr{Y}^{*}$ and both include $\mathscr{Y}^{*} \cap \mathscr{S}_{p}$.

(ii) $(\Rightarrow)$ We have

$$
\mathscr{Y} \in \Phi_{1} \Rightarrow \mathscr{H} \subseteq \mathscr{Y}+\mathscr{S}_{p} \Rightarrow \mathscr{H}+\mathscr{S}_{p} \subseteq \mathscr{Y}+\mathscr{S}_{p} ;
$$

therefore, intersecting both members with $y^{*}$, we obtain

$$
\mathscr{Y} * \cap\left(\mathscr{H}+\mathscr{S}_{p}\right) \subseteq \mathscr{Y} * \cap\left(\mathscr{Y}+\mathscr{S}_{p}\right)=\mathscr{Y}+\left(\mathscr{Y} * \cap \mathscr{S}_{p}\right)=\mathscr{Y},
$$

since $\left(\mathscr{Y}^{*} \cap \mathscr{S}_{p}\right) \subseteq\left(\mathscr{Y}^{*} \cap \mathscr{S}^{*}\right)$, which is the infimum of $\Phi$ and therefore is contained in all $\mathscr{Y} \in \Phi$.

(ii) $\quad(\Leftarrow)$ Note that

$$
\mathscr{Y} * \cap\left(\mathscr{H}+\mathscr{S}_{p}\right) \subseteq \mathscr{Y} .
$$

Adding $\mathscr{S}_{p}$ to both members, we obtain

$$
\mathscr{S}_{p}+\left(\mathscr{Y} * \cap\left(\mathscr{H}+\mathscr{S}_{p}\right)\right) \subseteq \mathscr{Y}+\mathscr{S}_{p},
$$

from which, using the distributive property, we obtain

$$
\left(\mathscr{S}_{p}+\mathscr{Y}^{*}\right) \cap\left(\mathscr{S}_{p}+\mathscr{H}\right) \subseteq \mathscr{Y}+\mathscr{S}_{p} ;
$$

since

$$
\mathscr{H} \subseteq \mathscr{S}_{p}+\mathscr{H} \subseteq \mathscr{S}_{p}+\mathscr{Y} *,
$$

we obtain

$$
\left(\mathscr{S}_{p}+\mathscr{H}\right) \subseteq \mathscr{Y}+\mathscr{S}_{p}
$$

and

$$
\mathscr{H} \subseteq \mathscr{Y}+\mathscr{S}_{p}
$$

Lemma 3.2. The infimum of $\Phi_{2}$ is given by

$$
\mathscr{q}_{m 2}=\mathscr{g} * \cap \min \mathscr{S}\left(A, \mathscr{Y} *, \mathscr{S}_{p}+\mathscr{H}\right) .
$$

Proof. The proof will be developed in two steps:
(A) Any element of $\Phi_{2}$ contains $\mathscr{Y}_{m 2}=\mathscr{y} * \cap \mathscr{S}_{2}^{*}$, where
$\mathscr{S}_{2}^{*}=\min \mathscr{S}\left(A, \mathscr{Y}^{*}, \mathscr{S}_{p}+\mathscr{H}\right)$;
(B) $\mathscr{y}^{*} \cap \mathscr{S}_{2}^{*}$ is an element of $\Phi_{2}$. 
Step A. Consider the sequence that defines $\mathscr{S}_{2}^{*}$,

$$
\begin{aligned}
& \mathscr{F}_{0}^{\prime}:=\mathscr{S}_{p}+\mathscr{H}, \\
& \mathscr{Z}_{i}^{\prime}:=\mathscr{S}_{p}+\mathscr{H}+A\left(\mathscr{F}_{i-1}^{\prime} \cap \mathscr{Y}^{*}\right), \quad i=1, \ldots
\end{aligned}
$$

Let $\mathscr{Y}$ be a generic element of $\Phi_{2}$, so that

$$
A \mathscr{Y} \subseteq \mathscr{Y}+\mathscr{B}, \quad \mathscr{Y} \supseteq \mathscr{Y} * \cap \mathscr{B} .
$$

We proceed by induction: Clearly,

$$
\mathscr{Z L}_{0}^{\prime} \cap \mathscr{*} \supseteq \mathscr{Y},
$$

since by assumption

$$
\mathscr{Y}^{*} \cap\left(\mathscr{S}_{p}+\mathscr{H}\right) \subseteq \mathscr{Y}
$$

and from

$$
\mathscr{Z}_{i-1}^{\prime} \cap \mathscr{Y} * \subseteq \mathscr{Y}
$$

it follows that

$$
A\left(\mathscr{Z}_{i-1}^{\prime} \cap \mathscr{Y} *\right) \subseteq A \mathscr{Y} \subseteq \mathscr{Y}+\mathscr{B},
$$

since $\mathscr{Y}$ is an $(A, \mathscr{B})$-controlled invariant. Adding $\mathscr{S}_{p}+\mathscr{H}$ to both members yields

$$
\mathscr{S}_{p}+\mathscr{H}+A\left(\mathscr{K}_{i-1}^{\prime} \cap \mathscr{Y}^{*}\right) \subseteq \mathscr{Y}+\mathscr{S}_{p}+\mathscr{H},
$$

where the left term of the last inclusion is by definition the subspace $\mathscr{Z}_{i}^{\prime}$ and, by intersecting with $\mathscr{Y}^{*}$, we obtain finally

$$
\mathscr{H}_{i}^{\prime} \cap \cap \mathscr{Y} * \subseteq\left(\mathscr{Y}+\left(\mathscr{S}_{p}+\mathscr{H}\right)\right) \cap \mathscr{Y} *=\mathscr{Y}+\left(\mathscr{S}_{p}+\mathscr{H}\right) \cap \mathscr{Y} *=\mathscr{F},
$$

which completes the induction argument and the proof of Step A.

Step B. It consists of proving the following statements:

(B1) $\mathscr{S} * \cap \mathscr{Y} *$ is an $(A, \mathscr{B})$-controlled invariant contained in $\mathscr{C}$,

(B2) $\mathscr{S}_{1}^{*} \cap \mathscr{Y} *$ is self-bounded with respect to $\mathscr{C}$,

(B3) $\mathscr{H} \subseteq\left(\mathscr{S}_{1}^{*} \cap \mathscr{Y}^{*}\right)+\mathscr{S}_{p}$,

from which it follows that $\mathscr{Y}^{*} \cap \mathscr{S}_{2}^{*}$ is an element of $\Phi_{2}$. 
To prove Statement B1, note that

$$
\begin{aligned}
& A \mathscr{Y}^{*} \subseteq \mathscr{Y} *+\mathscr{B}, \\
& A\left(\mathscr{S}_{1}^{*} \cap \mathscr{Y} *\right) \subseteq \mathscr{S}^{*},
\end{aligned}
$$

which express simply $\mathscr{Y} *$ as an $(A, \mathscr{B})$-controlled invariant and $\mathscr{S}_{1}^{*}$ as an $\left(A, \mathscr{Y}^{*}\right)$-conditioned invariant. By intersection, it follows that

$$
A\left(\mathscr{S}_{1}^{*} \cap \mathscr{Y}^{*}\right) \subseteq \mathscr{S}_{1}^{*} \cap\left(\mathscr{Y}^{*}+\mathscr{B}\right)=\mathscr{S}_{1}^{*} \cap \mathscr{Y} *+\mathscr{B},
$$

since $\mathscr{B} \subseteq \mathscr{S}_{1}^{*}$. Then, $\mathscr{S}_{1}^{*} \cap \mathscr{Y} *$ is an $(A, \mathscr{B})$-controlled invariant contained in $\mathscr{C}$.

To prove Statement B2, note that

$$
\mathscr{Y} * \cap \mathscr{B} \subseteq \mathscr{Y} * \cap \mathscr{S}_{1}^{*} .
$$

Finally, to prove Statement B3, note that, since

$$
\mathscr{S}_{p} \subseteq \mathscr{S}_{1}^{*} \text { and } \mathscr{H} \subseteq \mathscr{S}_{1}^{*} \text {, }
$$

it follows that

$$
\mathscr{H} \subseteq\left(\mathscr{Y}^{*} \cap \mathscr{S}_{p}\right)+\mathscr{S}_{i}^{*}=\left(\mathscr{Y} * \cap \mathscr{S}_{i}^{*}\right)+\mathscr{S}_{p} .
$$

Lemma 3.3. The set

$$
\Phi_{3}=\left\{\mathscr{V} \mid \mathscr{Y} \in \Phi, \mathscr{K}_{V} \subseteq \mathscr{Y}+\mathscr{S}_{p}\right\}
$$

enjoys the following properties:

(i) it is a sublattice of $\Phi$;

(ii) $\Phi_{3} \equiv\left\{\mathscr{Y} \mid \mathscr{Y} \in \Phi, \mathscr{Y} * \cap\left(\mathscr{H}_{V}+\mathscr{S}_{p}\right) \subseteq \mathscr{Y}\right\}$;

(iii) the infimum of $\Phi_{3}$ is given by

$$
\mathscr{y}_{m 3}=\mathscr{y}^{*} \cap \min \mathscr{C}\left(A, \mathscr{y}^{*}, \mathscr{S}_{p}+\mathscr{H}_{V}\right) ;
$$

(iv) $\Phi_{3} \equiv \Phi_{2}$; i.e., $\mathscr{\mathscr { T }}_{\mathrm{m} 2} \equiv \mathscr{q}_{\mathrm{m} 3}$;

(v) $\Phi_{3} \subseteq \Phi_{1}$;

(vi) $\mathscr{\mathscr { T }}_{m 1} \in \Phi_{3}$;

(vii) $\mathscr{C}_{m 1} \equiv \mathscr{V}_{m 2} \equiv \mathscr{V}_{m 3}$. 


\section{Proof.}

(i) to (iii). From (8), it follows that

$$
\mathscr{H}_{V} \subseteq \mathscr{Y} *+\mathscr{S}_{p} .
$$

Therefore, the proofs of Properties (i) and (ii) are analogous to those of Properties (i), (ii) in Lemma 3.1, while the proof of Property (iii) is similar to that in Lemma 3.2.

(iv) This property follows from Property (ii) of this statement, Lemma 3.1, and Eqs. (7) and (9).

(v) Let $\mathscr{V} \in \Phi_{3}$. It follows that:

$$
\begin{aligned}
& A \mathscr{Y} \subseteq \mathscr{Y}+\mathscr{B}+\mathscr{H}_{V}, \text { since } \mathscr{Y} \text { is an }(A, \mathscr{B}) \text {-controlled invariant, } \\
& \mathscr{Y} \subseteq \mathscr{C}, \\
& \mathscr{Y} * \cap\left(\mathscr{B}+\mathscr{H}_{V}\right) \subseteq \mathscr{Y} * \cap\left(\mathscr{S}_{p}+\mathscr{H}_{V}\right) \subseteq \mathscr{Y} .
\end{aligned}
$$

Then,

$$
\mathscr{V} \in \Phi_{3} \Rightarrow \mathscr{V} \in \Phi_{1} .
$$

(vi) First of all, note that

$$
A \mathscr{Y}_{m 1} \subseteq \mathscr{Y}_{m 1}+\mathscr{B},
$$

since

$$
\begin{aligned}
A^{\mathscr{Y}}{ }_{m 1} & =A((\mathscr{Y} * \cap \mathscr{C}) \cap \min \mathscr{S}(A, \mathscr{C}, \mathscr{B}+\mathscr{C} V)) \\
& \subseteq A \mathscr{Y}^{*} \cap A(\mathscr{C} \cap \min \mathscr{(}(A, \mathscr{C}, \mathscr{B}+\mathscr{C} V)) \\
& \subseteq\left(\mathscr{Y}^{*}+\mathscr{B}\right) \cap \min \mathscr{S}\left(A, \mathscr{C}, \mathscr{B}+\mathscr{C}_{V}\right) \\
& =\left(\mathscr{Y} *<3 r \cap \min \mathscr{C}\left(A, \mathscr{C}, \mathscr{B}+\mathscr{C}_{V}\right)\right)+\mathscr{B} \\
& =\mathscr{Y}_{m 1}+\mathscr{B} ;
\end{aligned}
$$

moreover,

$$
\mathscr{Y}_{m 1} \subseteq \mathscr{C} \text { and } \mathscr{Y}^{*} \cap \mathscr{B} \subseteq \mathscr{Y}_{m 1} ;
$$

then, $\mathscr{Y}_{m 1} \in \Phi$. Finally, note that

$$
\mathscr{H}_{V} \subseteq \mathscr{Y}_{m 1}+\mathscr{S}_{p} .
$$

In fact, since

$$
\mathscr{S}_{p} \subseteq \min \mathscr{S}\left(A, \mathscr{C}, \mathscr{B}+\mathscr{H}_{V}\right),
$$


we have

$$
\begin{aligned}
\mathscr{Y}_{m 1}+\mathscr{S}_{p} & =(\mathscr{Y} * \cap \min \mathscr{A}(A, \mathscr{C}, \mathscr{B}+\mathscr{H} V))+\mathscr{S}_{p} \\
& =\left(\mathscr{Y} *+\mathscr{S}_{p}\right) \cap \min \mathscr{S}\left(A, \mathscr{C}, \mathscr{B}+\mathscr{H}_{V}\right),
\end{aligned}
$$

and $\mathscr{H}_{V}$ is included in both the subspaces of the latter intersection.

(vii) It is implied by Properties (iv)-(vi).

Before stating the main result, let us recall a fundamental property, proved in Ref. 12, of self-bounded subspaces.

Remark 3.1. Let $\mathscr{T}$ and $\mathscr{Y}$ be a pair of $(A, \mathscr{B})$-controlled invariant subspaces self-bounded with respect to $\mathscr{C}$ [see Eq. (11)], such that $\mathscr{V} \subseteq \mathscr{Y}$. Let $F$ be a matrix such that $(A+B F) \subseteq \mathscr{Y}$. Then, $(A+B F) \mathscr{Y}$.

Theorem 3.1. The signal decoupling problem with stability for the $p$ previewed signal ${ }^{p} h(k)$ stated in Problem P2 is solvable if and only if the structural condition (4) is satisfied and

$$
\mathscr{Y}_{m 1}=\mathscr{Y} * \cap \min \mathscr{S}(A, \mathscr{C}, \mathscr{B}+\mathscr{H} V),
$$

defined in (13), is internally stabilizable.

Proof. As shown in Theorem 2.1, the purpose of the preaction unit is to cancel, at the generic time instant $k$, the component of $H h(k)$ on $\mathscr{S}_{p}$ in order to force the state dynamics [excited by the other component $H_{V} h(t)$ ] on a subspace $\mathscr{V}$ satisfying the following properties:

(i) $\mathscr{Y}$ is an $(A, \mathscr{B})$-controlled invariant included in $\mathscr{C}$;

(ii) $\mathscr{Y}$ is such that $\mathscr{H} \subseteq \mathscr{Y}+\mathscr{S}_{p}$;

(iii) $\mathscr{Y}$ is internally stabilizable.

We will now prove the necessity of the statement; i.e., if a subspace $\mathscr{Y}$ exists that solves Problem P2 with stability, then $\int_{m 1}$ is internally stabilizable. Since $\mathscr{y}_{m 1}=\mathscr{y}_{m 2}\left(\right.$ Lemma 3.3), we will refer henceforth to $\mathscr{y}_{m 2}$. Consider the subspace

$$
\mathscr{V}:=\mathscr{V}+\mathscr{R}_{7} *
$$

where $\mathscr{y}$ is a subspace satisfying Properties (i)-(iii) and $\mathscr{R}^{*}=\mathscr{Y}^{*} \cap \mathscr{S}^{*}$ represents the constrained reachability subspace on $\mathscr{C}$. It 
is clear that $\bar{s}$ satisfies Properties (i)-(iii) because:

$\mathscr{F}$ is an $(A, \mathscr{B})$ controlled invariant contained in $\mathscr{C}$, since it is the sum of two controlled invariants contained in $\mathscr{C}$;

$\mathscr{H} \subseteq \overline{\mathscr{Y}}+\mathscr{S}_{p}$, since $\mathscr{H} \subseteq \mathscr{Y}+\mathscr{S}_{p}$;

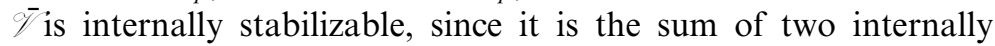
stabilizable subspaces.

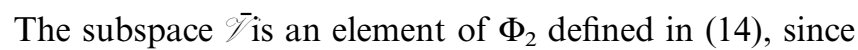

$$
\mathscr{Q} * \cap \mathscr{B} \subseteq \mathscr{R}, \subseteq \%
$$

Because $\bar{Y}$ is internally stabilizable, a state feedback matrix $F$ exists that stabilizes such subspace. Because of Remark 3.1, such matrix stabilizes every subspace $\mathscr{Y} \in \Phi_{2}$ included in $\mathscr{\%}$, and therefore also its infimum $\mathscr{Y} \mathrm{m}$, since all of these subspaces are self-bounded.

For the sufficiency part, simply note that, if $\mathscr{q}_{m 1}$ is internally stabilizable, then it satisfies Properties (i)-(iii) at once.

Regarding the dimension of the resolving subspace, it can be shown easily that

$$
\mathscr{Y}_{m} \subseteq \mathscr{Y}_{g},
$$

where $\mathscr{Y}_{g}$ is the resolving subspace defined in Ref. 4. In fact, since $\mathscr{R}_{j} \subseteq_{\mathrm{g}}$, from the proof of Theorem 3.1, it follows that

$$
\mathscr{P}=\mathscr{q}_{g}+\mathscr{R}_{*} * \mathscr{\mathscr { V }}_{\mathrm{g}} \in \Phi_{2} \text {, }
$$

whose infimum is $\mathscr{Y}_{\mathrm{m}}$.

The second main contribution of this paper consists in unifying the stability condition for the disturbance decoupling problem, measurable signal, decoupling problem, reported in Ref. 12, and the more general previewed signal decoupling problem.

Theorem 3.2. The signal decoupling problem with stability for the $p$ previewed signal ${ }^{p} h(k)$ stated in Problem P2 is solvable if and only if the structural condition (4) is satisfied and

$$
\mathscr{C}_{m}:=\mathscr{Y} * \cap \min \mathscr{A}(A, \mathscr{C}, \mathscr{B}+\mathscr{H})
$$

is internally stabilizable.

Proof. The proof is based on Theorem 3.1 and on the equivalence

$$
\mathscr{Y}_{m 1} \equiv \mathscr{Y}_{m},
$$


which is now proved. Since

$$
\mathscr{H}_{S} \subseteq \mathscr{S}_{p} \subseteq \min \mathscr{S}(A, \mathscr{C}, \mathscr{B}) \subseteq \min \mathscr{S}(A, \mathscr{C}, \mathscr{B}+\mathscr{C} V),
$$

from (7) we have

$$
\begin{aligned}
& \mathscr{B}+\mathscr{H} \subseteq \min \mathscr{S}(A, \mathscr{C}, \mathscr{B}+\mathscr{H} V), \\
& \mathscr{B}+\mathscr{H}_{V} \subseteq \min \mathscr{S}(A, \mathscr{C}, \mathscr{B}+\mathscr{H}) .
\end{aligned}
$$

Now, let us prove that

$$
\min \mathscr{S}(A, \mathscr{C}, \mathscr{B}+\mathscr{H}) \subseteq \min \mathscr{S}(A, \mathscr{C}, \mathscr{B}+\mathscr{H} V),
$$

hence by applying induction arguments to the subspace sequence defining $\min \mathscr{S}(A, \mathscr{C}, \mathscr{B}+\mathscr{H})$, whose $i$ th element is $\mathscr{Z}^{\prime}{ }^{\prime}$. From (27), one gets

$$
\mathscr{Z}_{0}^{\prime}=\mathscr{B}+\mathscr{H} \subseteq \min \mathscr{S}(A, \mathscr{C}, \mathscr{B}+\mathscr{H} V) .
$$

Assume that

$$
\mathscr{Z}_{i-1}^{\prime} \subseteq \min \mathscr{S}\left(A, \mathscr{C}, \mathscr{B}+\mathscr{C}_{V}\right) .
$$

Since min $\mathscr{S}(A, \mathscr{C}, \mathscr{B}+\mathscr{H} V)$ is an $(A, \mathscr{C})$-conditioned invariant containing $\mathscr{B}+\mathscr{H}$, it follows that

$$
\mathscr{B}+\mathscr{H}+A\left(\mathscr{H}_{i-1}^{\prime} \cap \mathscr{C}\right) \subseteq \min \mathscr{S}\left(A, \mathscr{C}, \mathscr{B}+\mathscr{H}_{V}\right) .
$$

Similarly, starting from (28), it is possible to prove that

$$
\min \mathscr{S}(A, \mathscr{C}, \mathscr{B}+\mathscr{H} V) \subseteq \min \mathscr{S}(A, \mathscr{C}, \mathscr{B}+\mathscr{H}) .
$$

Finally, from (29) and (30), one gets

$$
\min \mathscr{S}(A, \mathscr{C}, \mathscr{B}+\mathscr{H} V)=\min \mathscr{S}(A, \mathscr{C}, \mathscr{B}+\mathscr{H}),
$$

and the equivalence (26) is proven.

Remark 3.2. The necessary and sufficient conditions for the disturbance decoupling problem (DDP), measurable signal decoupling problem (MSPD), and previewed signal decoupling problem (PSDP) with stability differ only for the structural conditions (6), (5), (4) respectively, but not for the stability condition, which is unique and corresponds to the internal stabilizability of the controlled invariant $y_{m}$ [see (25)]. 


\section{Synthesis Procedure for the Controller Solving the PSDP}

Assume that the necessary and sufficient conditions of Theorem 3.1 or Theorem 3.2 hold. Due to Eq. (7), it is possible always to decompose the effect of $H h(k)$ on the system dynamics into two separate parts. The effect of $H_{V} \mathrm{~h}(k)$ can be decoupled using a postaction unit, since $\mathscr{H}_{V} \subseteq \mathscr{Y}^{*}$.

The effect of $H_{S} h(k)$ can be nulled using a preaction unit, since $\mathscr{H}_{s} \subseteq \mathscr{S}_{p}$.

4.1. Postaction Unit. Due to (7), the system (1) can be rewritten as

$$
\begin{aligned}
& x(k+1)=A x(k)+B u(k)+H_{V} h(k)+H_{S} h(k), \\
& y(k)=C x(k),
\end{aligned}
$$

where

$$
u(k)=u_{\mathrm{pos}}(k)+u_{\mathrm{pre}}(k) .
$$

The purpose of the postaction unit is to decouple $\mathscr{H}_{v}$. It is an easy matter to show that $\mathscr{H}_{V} \subseteq \mathscr{Y}_{m 1}$. Applying Theorem 3.1, a stabilizing state feedback matrix $F$ exists such that the state trajectory excited by $H_{V} h(k)$ evolve onto $\mathscr{Y}_{m 1} \in \mathscr{C}$. Therefore, the postaction unit is simply given by

$$
u_{\text {pos }}(k)=F x(k) \text {, }
$$

as shown in Fig. 1.

It is worth noting that, since the system dynamics evolve on a known subspace, the postaction unit can be implemented also as a feedforward unit.

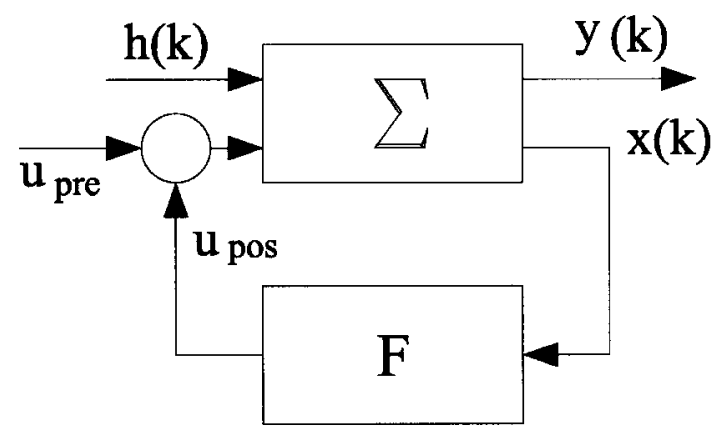

Fig. 1. Postaction control $u_{\text {pos }}(k)$ synthesized through a state feedback matrix $F$. 
4.2. Preaction Unit. In order to design the preaction unit, the subspace $\mathscr{S}_{p}$ must be interpreted as a special reachability subspace.

Property Q2. The subspace $\mathscr{S}_{p}$ corresponds to the set of states reachable in $p, p \geq 0$, steps from the initial condition $x_{0}=0$, with the state trajectory constrained to evolve onto $\mathscr{C}$ in the preceding $p$-step interval $[0, p-1]$.Analytically, matrices $\Omega_{0}, \Omega_{1}, \ldots, \Omega_{p}$ exist such that

$$
\begin{gathered}
\mathscr{S}_{p}=\operatorname{im}\left[B, A B, A^{2} B, \ldots, A^{p} B\right]\left[\begin{array}{l}
\Omega_{0} \\
\Omega_{1} \\
\Omega_{2} \\
\vdots \\
\Omega_{p}
\end{array}\right], \\
{\left[\begin{array}{llll}
C B & C A B & \ldots & C A^{p-1} B \\
0 & C B & \ldots & C A^{p-2} B \\
\vdots & \vdots & \ddots & \vdots \\
0 & \ldots & 0 & C B
\end{array}\right]\left[\begin{array}{l}
\Omega_{1} \\
\Omega_{2} \\
\vdots \\
\Omega_{p}
\end{array}\right]=\left[\begin{array}{l}
0 \\
0 \\
\vdots \\
0
\end{array}\right] .}
\end{gathered}
$$

Taking into account the postaction unit (32), the system (31) can be rewritten as

$$
x(k+1)=A_{f} x(k)+B u_{\mathrm{pre}}(k)+H_{s} h(k)+H_{v} h(k),
$$

where

$$
A_{f}:=A+B F .
$$

To decouple the effects of $H_{S} h(k)$ on the system dynamics (33), a preaction unit is built as

$$
u_{\mathrm{pre}}(k)=\sum_{l=0}^{p} \Phi(l) h(k+l),
$$

where the gains of the preaction unit are computed as

$$
\left[\begin{array}{c}
\Phi_{0} \\
\Phi_{1} \\
\vdots \\
\Phi_{p}
\end{array}\right]=M^{\#}\left[\begin{array}{c}
-H_{s} \\
0 \\
0 \\
\vdots \\
0
\end{array}\right] .
$$




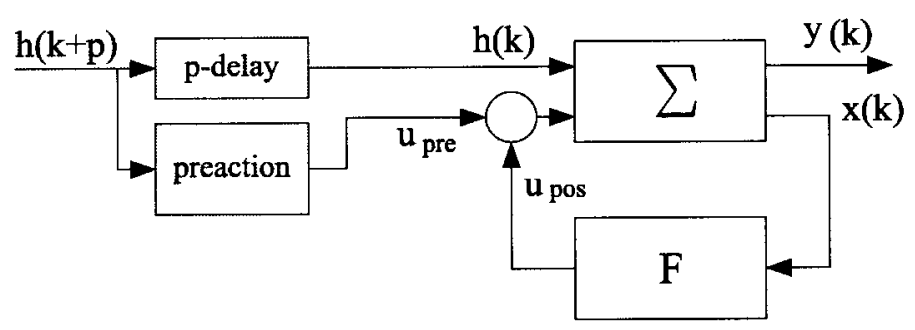

Fig. 2. Decoupling compensator consisting of a preaction unit and a postaction unit.

\section{Since}

$$
M=\left[\begin{array}{llllc}
B & A_{f} B & A_{f}^{2} B & \ldots & A_{f}^{p} B \\
0 & C B & C A_{f} B & \ldots & C A_{f}^{p-1} B \\
0 & 0 & C B & \ldots & C A_{f}^{p-2} B \\
\vdots & \vdots & \vdots & \ddots & \vdots \\
0 & 0 & \ldots & 0 & C B
\end{array}\right]
$$

the consistency of (35) is guaranteed by Property Q2 and the inclusion (9).

The preaction unit in Eq. (34) is shown in Fig. 2. Preaction consists of a $p$-step FIR system which, previewing the signal $h(k) p$-steps in advance, is able to prepare the system dynamics to cancel the component $H_{S} h(k)$, when it presents itself as input to the system at the time instant $k$.

\section{Illustrative Example}

Let us consider the previewed signal decoupling problem for the system (1) with

$$
A=0.1\left[\begin{array}{rrrrr}
1 & 2 & 1 & -1 & -2 \\
0 & -1 & 2 & 1 & 1 \\
0 & 3 & 1 & -1 & -1 \\
1 & 1 & 2 & 2 & 1 \\
0 & 0 & 0 & 1 & -5
\end{array}\right], \quad B=\left[\begin{array}{ll}
1 & 0 \\
0 & 0 \\
0 & 0 \\
0 & 1 \\
0 & 0
\end{array}\right]
$$




$$
H=\left[\begin{array}{ccc}
1 & 0 & 0 \\
0 & 0 & 0.5774 \\
0 & 0 & -0.5774 \\
0 & 1 & 0 \\
0 & 0 & 0.5774
\end{array}\right], \quad C=[0,0,0,0,1]
$$

The algorithms for $\mathscr{f} *$ and $\mathscr{S}^{*}$ converge to

$$
\mathscr{Y} *=\operatorname{im}\left[\begin{array}{ccc}
1 & 0 & 0 \\
0 & 1 & 0 \\
0 & 0 & 1 \\
0 & 0 & 0 \\
0 & 0 & 0
\end{array}\right], \quad \mathscr{S}^{*}=\operatorname{im}\left[\begin{array}{ccc}
1 & 0 & 0 \\
0 & 0 & 0.5774 \\
0 & 0 & -0.5774 \\
0 & 1 & 0 \\
0 & 0 & 0.5774
\end{array}\right] .
$$

Note that

$$
\mathscr{S}_{1}=\mathscr{S}^{*}
$$

and that

$$
\mathscr{H} \subseteq \mathscr{Y}^{*}+\mathscr{S}_{1}=\mathscr{Y} *+\mathscr{S}^{*} .
$$

Since

$$
\mathscr{H} \nsubseteq \mathscr{y} *+\mathscr{B},
$$

$p=1$ is the minimum number of previewed steps necessary to solve the decoupling problem.

According to (7), the matrix $H$ is decomposed in the two matrices

$$
H_{V}=\left[\begin{array}{lll}
1 & 0 & 0 \\
0 & 0 & 0 \\
0 & 0 & 0 \\
0 & 0 & 0 \\
0 & 0 & 0
\end{array}\right], \quad H_{S}=\left[\begin{array}{ccc}
0 & 0 & 0 \\
0 & 0 & 0.5774 \\
0 & 0 & -0.5774 \\
0 & 1 & 0 \\
0 & 0 & 0.5774
\end{array}\right] .
$$

The resolving subspace is evaluated as

$$
\mathscr{Y}_{m 1}=\mathscr{Y}_{m 2}=\operatorname{im}\left[\begin{array}{l}
1 \\
0 \\
0 \\
0 \\
0
\end{array}\right]
$$

which is internally stabilizable. 
It is worth noting that subspace $\mathscr{Y}_{g}^{*}$ proposed in Ref. 4 has, in this case, a dimension which is double that of $\mathscr{9} \mathrm{m}$.

The postaction unit is given by the state feedback

$$
u_{\mathrm{pos}}=F x(k),
$$

where

$$
F=\left[\begin{array}{ccccc}
-0.1 & 0 & 0 & 0.1 & 0 \\
-0.1 & 0 & 0 & -0.2 & 0
\end{array}\right],
$$

while for the preaction unit, we obtain from (35) and (36)

$$
\Phi_{1}=\left[\begin{array}{ccc}
0 & 0 & 0 \\
0 & 0 & -5.7735
\end{array}\right], \quad \Phi_{0}=\left[\begin{array}{ccc}
0 & 0 & 0 \\
0 & 1 & 0
\end{array}\right] .
$$

Simulations are executed by applying a signal

$$
h(k)=\left[h_{1}(k), h_{2}(k), h_{3}(k)\right]^{T},
$$

where $h_{1}(k), h_{2}(k), h_{3}(k)$ are band-limited white noises, as shown in Fig. 3.

The two components of the postaction and preaction signals $u_{\text {pos }}(k)$ are shown in Fig. 4 and Fig. 5, respectively. The preaction-postaction compensator decouples perfectly the signal $h(k)$ which has a 1-step preview and maintains the state trajectory bounded.

\section{Conclusions}

A new solution for general signal decoupling problems with stability has been proposed. It is based on two necessary and sufficient constructive conditions: one is structural in nature, while the other deals with the stability requirement. The problem has been approached through self-bounded controlled invariants, thus allowing one to reduce the dimension of the resolving subspace which corresponds to the infimum of a lattice.

It has been shown that the conditions to be solved for the decoupling problem with stability apply independently of the type of signal to be decoupled, no matter whether it is completely unknown, measured, or previewed. In other terms, each problem specializes only in its structural condition. The stability condition is the same for the DDP, MSDP, and PSDP, since the resolving subspace, whose internal stabilizability needs to be checked, is the infimum of the same lattice.

Finally, a new and easily implementable synthesis procedure has been proposed and used to solve a simple example. 

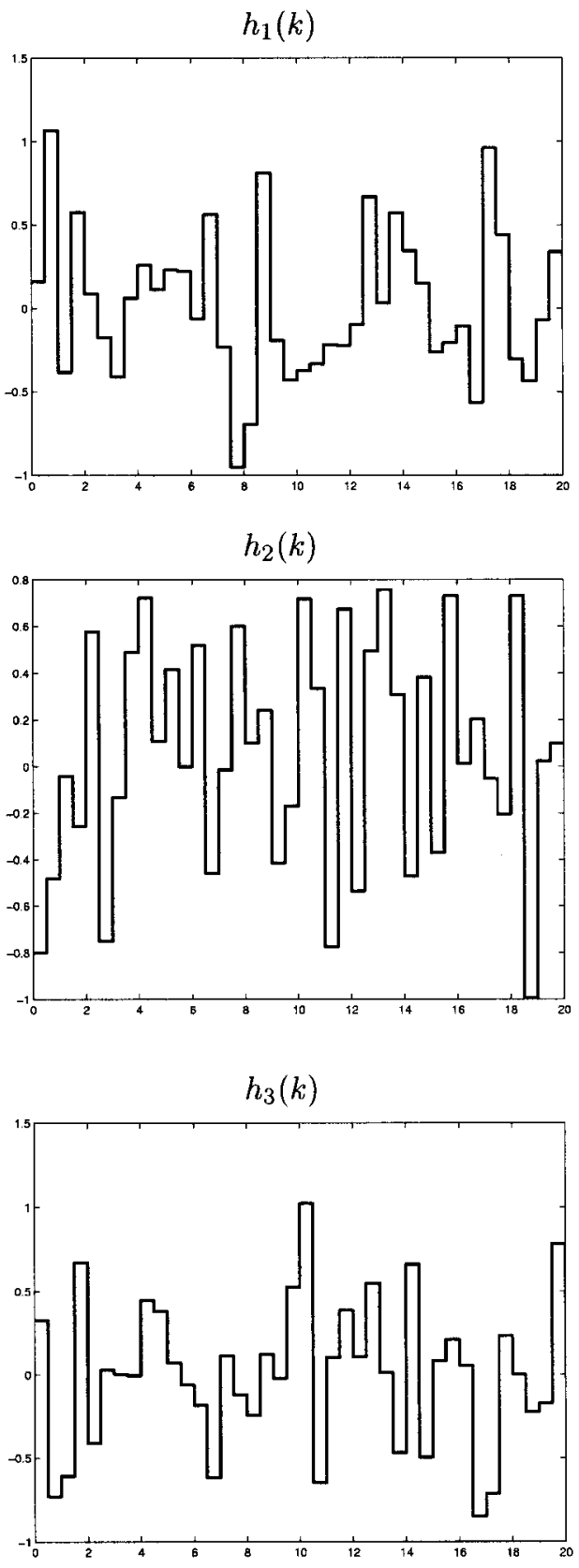

Fig. 3. Components of the 1-previewed signal $h(k)$. 


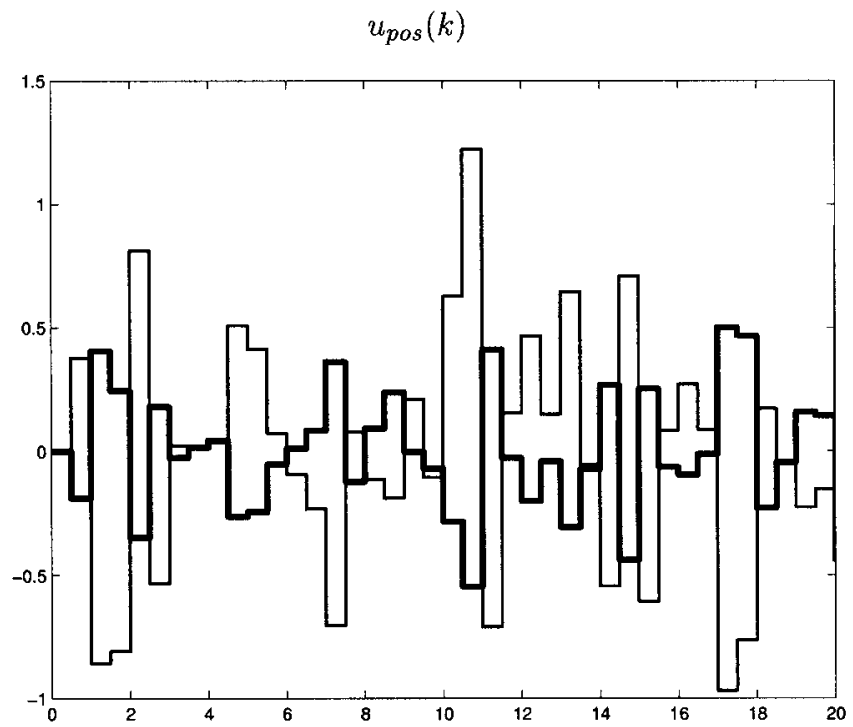

Fig. 4. Components of the postaction control $u_{\text {pos }}(k)$.

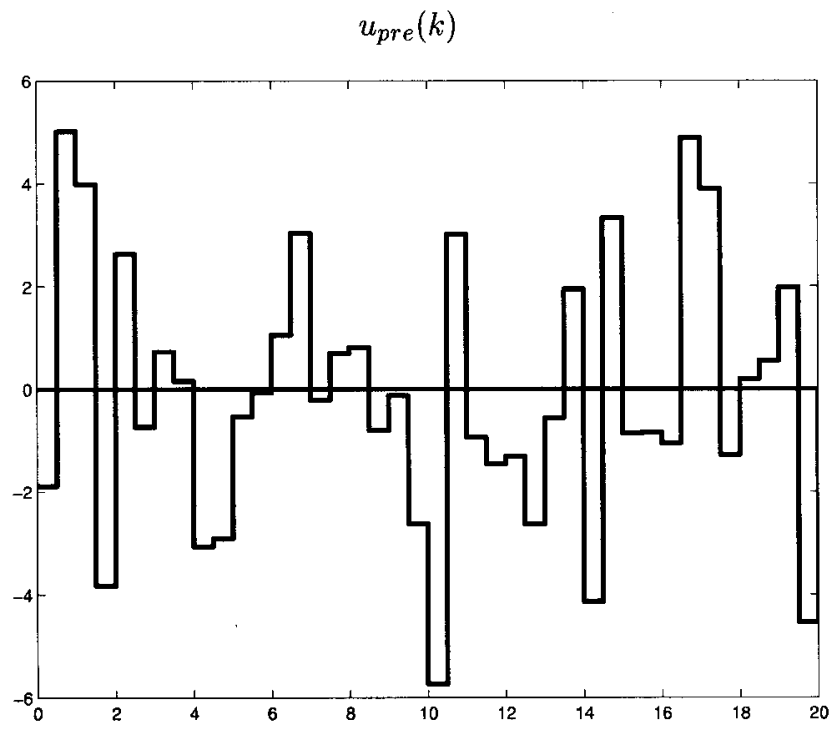

Fig. 5. Components of the preaction control $u_{\text {pre }}(k)$. 


\section{References}

1. Basile, G., and Marro, G., Disturbance Decoupling Considered in the State Space, Paper 1-4-01, 70th Annual AEI Meeting, Rimini, Italy, 1969 (in Italian).

2. Wonham, W. M., and Morse, A. S., Decoupling and Pole Assignment in Linear Multivariable Systems: A Geometric Approach, SIAM Journal on Control and Optimization, Vol. 8, No. 1, pp. 1-18, 1970.

3. Bhattacharyya, S. P., Disturbance Rejection in Linear Systems, International Journal of Systems Science, Vol. 5, No. 7, pp. 931-943, 1974.

4. Willems, J. C., Feedforward Control, PID Control Laws, and Almost Invariant Subspaces, Systems and Control Letters, Vol. 1, No. 4, pp. 277-282, 1982.

5. Imai, H., Shinozuka, M., Yamaki, T., Li, D., and Kuwanasile, M., Disturbance Decoupling by Feedforward and Preview Control, Journal of Dynamic Systems, Measurement, and Control, Vol. 105, No. 11, pp. 931-943, 1983.

6. Estrada, M. B., and Malabre, M., Necessary and Sufficient Conditions for Disturbance Decoupling with Stability Using PID Control Laws, IEEE Transaction on Automatic Control, Vol. 44, No. 6, pp. 1311-1315, 1999.

7. Basile, G., and Marro, G., Self-Bounded Controlled Subspaces: A Straightforward Approach to Constrained Controllability, Journal of Optimization Theory and Applications, Vol. 38, No. 1, pp. 71-81, 1982.

8. Schumacher, J. M., On a Conjecture of Basile and Marro, Journal of Optimization Theory and Applications, Vol. 41, No. 2, pp. 371-376, 1983.

9. Basile, G., Marro, G., and Piazzi, A., A New Solution to the Disturbance Localization Problem with Stability and Its Dual, Proceedings of the 1984 AMSE International Conference on Modelling and Simulation, Athens, Greece, Vol. 1.2, pp. 19-27, 1984.

10. Barbagli, F., Marro, G., and Prattichizzo, D., Previewed Signal Decoupling Problem in Discrete-Time Systems, Proceedings of MTNS 2000, Perpignan, France, 2000.

11. Barbagli, F., Marro, G., and Prattichizzo, D., Solving Signal Decoupling Problems through Self-Bounded Controlled Invariants, Proceedings of the 39th IEEE Conference on Decision and Control, Sydney, Australia, 2000.

12. Basile, G., and Marro, G., Controlled and Conditioned Invariants in Linear System Theory, Prentice-Hall, Englewood Cliffs, New Jersey, 1992. 